\title{
A NOTE ON INTEGRAL REPRESENTATION OF SOME GENERALIZED ZETA FUNCTIONS AND ITS CONSEQUENCES
}

\author{
KHALED MEHREZ
}

Abstract. The main focus of the present note is to establish new integral representation for the Hurwitz-Lerch zeta and the multi-parameter Hurwitz-Lerch zeta functions. In particular, new integral expression of the polylogarithm function and the Fox-Wright function are derived. In addition, closed integral form expression of the moment generating function of a zeta distribution is established. As application, we derive the complete monotonicity properties of two classes of function related to the Hurwitz-Lerch zeta and the polylogarithm function. Moreover, some inequalities involving these two functions are proved.

Mathematics subject classification (2010): 62M10, 40C10, 62M20, 33C10.

Keywords and phrases: Hurwitz-Lerch zeta function, Fox-Wright function, Cahen integral, Dirichlet series.

\section{REFERENCES}

[1] E. CAHEN, Sur la fonction $\zeta(s)$ de Riemann et sur des fontions analogues, Ann Sci l'École Norm Sup Ser Math., 11 (1894), 75-164.

[2] A. Erdélyi, W. Magnus, F. Oberhettinger, F. G Tricomi, Higher transcendental functions, vol. I. McGraw-Hill Book Company, New York (1953).

[3] A. Erdélyi, W. Magnus, F. Oberhettinger, F. Tricomi, Tables of Integral Transforms, vol. I, McGraw-Hill Book Company, New York, Toronto and London, (1954).

[4] S. P. GOYAL, R. K. LADDHA, On the generalized Riemann zeta function and the generalized Lambert transform, Ganita Sandesh, 11 (1997), 99-108.

[5] M. GARG, K. JAIN, S. L. KALLA, A further study of general Hurwitz-Lerch zeta function, Algebras Groups Geom., 25 (2008), 311-319.

[6] D. Jankov, T. K. PogÁny, R. K. SAXena, Extended general Hurwitz-Lerch Zeta function as Mathieu $(a, \lambda)$-series, Appl. Math. Lett., (24), 8 (2011), 1473-1476.

[7] C. H. Kimberling, A probabilistic interpretation of complete monotonicity, Aequationes Math., 10 (1974), 152-164.

[8] K. MehreZ, New Integral representations for the Fox-Wright functions and its applications, J. Math. Anal. Appl., 468 (2018), 650-673.

[9] K. MenreZ, New properties for several classes of functions related to the Fox-Wright functions, Journal of Computational and Applied Mathematics, 362 (2019), 161-171.

[10] S. Mizukami, T. Nakamura, Generalized Hurwitz zeta distributions, Siauliai Math. Semin., 8, 16 (2013), 151-160.

[11] O. X. Perron, Zur Theorie der Dirichletschen Reihen, J. Reine Angew Math., 134 (1908), 95-143.

[12] T. K. PogÁNY, Integral form of the COM-Poisson renormalization constant, Statist Probab Lett. 119 (2016), 144-145.

[13] T. K. PogÁnY, Integral form of Le Roy-type hypergeometric function, Integral Transforms Spec. Funct., 29, 7 (2018), 580-584.

[14] T. K. PogÁny, Integral representation of Mathieu $(a, \lambda)$-series, Integral Transforms Spec Funct., 16, 8 (2005), 685-689.

[15] T. K. PogÁNY, E. SÜLI, Integral representation for Neumann series of Bessel functions, Proc. Amer. Math. Soc., 137, 7 (2009), 2363-2368. 
[16] H. M. SRivastava, R. K. Saxena, T. K. Pogány, R. Saxena, Integral and computational representations of the extended Hurwitz-Lerch zeta function, Integral Transforms Spec. Funct., 22 (2011), 487-506.

[17] H. M. SRivastava, J. Choi, Series Associated with Zeta and Related Functions, Kluwer Academic, Dordrecht (2001).

[18] D. V. WidDER, The Laplace Transform, Princeton Univ. Press, Princeton, 1941.

[19] E. M. WRIGHT, The asymptotic expansion of the generalized hypergeometric function, Journal London Math. Soc., 10 (1935), 287-293. 